\title{
Antiepileptic drug treatment in the end-of-life phase of glioma patients: a feasibility study
}

\author{
Johan A. F. Koekkoek ${ }^{1,2,3}$ - Tjeerd J. Postma ${ }^{1}$. Jan J. Heimans ${ }^{1}$ - Jaap C. Reijneveld ${ }^{1}$. \\ Martin J. B. Taphoorn ${ }^{1,2,3}$
}

Received: 7 April 2015 / Accepted: 31 August 2015 / Published online: 25 September 2015

(C) The Author(s) 2015. This article is published with open access at Springerlink.com

\begin{abstract}
Background During the end-of-life (EOL) phase of glioma patients, a rapid deterioration in neurological functioning may interfere with the oral intake of antiepileptic drugs (AEDs). We aimed to assess the feasibility of non-oral AED treatment in an out-of-hospital setting according to an expert-based guideline. Methods Glioma patients with a history of epilepsy, in whom further antitumor therapy was considered to be no longer meaningful, were recruited at two Dutch hospitals. As soon as swallowing difficulties developed, the patient's caregiver administered prophylactic treatment with buccal clonazepam. Acute seizures were treated with intranasal midazolam. We evaluated the adherence to the study medication, seizure prevalence, and caregiver's satisfaction.

Results Of the 34 patients who were approached, 25 gave consent to participate and 23 had died at the end of the study. Thirteen of 19 patients $(68.4 \%)$ who had developed swallowing difficulties showed adherence to the study protocol. Thirteen patients used prophylactic buccal clonazepam, of which eight patients remained seizure-free until death. Six patients received treatment with intranasal midazolam at least
\end{abstract}

Electronic supplementary material The online version of this article (doi:10.1007/s00520-015-2930-3) contains supplementary material, which is available to authorized users.

Johan A. F. Koekkoek

j.koekkoek@vumc.nl

1 Department of Neurology, VU University Medical Center, PO Box 7057, 1007 MB Amsterdam, The Netherlands

2 Department of Neurology, Medical Center Haaglanden, PO Box 432, 2501 CK The Hague, The Netherlands

3 Department of Neurology, Leiden University Medical Center, PO Box 9600, 2300 RC Leiden, The Netherlands once. In all patients, seizure control was reached. None of the patients needed to be transferred to the hospital due to recurrent seizures. All caregivers were to some degree satisfied with the use of the study medication.

Conclusions Our results demonstrate that it is feasible to treat seizures with a combination of non-oral benzodiazepines in the EOL phase of glioma patients, as it seems to provide an important level of comfort among caregivers to be able to manage seizures at home.

Keywords Glioma · End of life · Midazolam · Clonazepam · Antiepileptic drugs $\cdot$ Epilepsy

\section{Introduction}

Seizures are a relatively common symptom in patients with a glioma, occurring in 30-90\% of patients, depending on the glioma subtype [1,2]. Recent studies have shown that seizures persist during the end stage of the disease in many glioma patients, with reported prevalence during the end-of-life (EOL) phase ranging from 21 to $52 \%$ [3-5]. Patients who have a history of epilepsy are particularly prone to suffer from seizures in the EOL phase, but de novo seizures in the EOL phase have also been reported $[3,6]$.

During the EOL phase, care is primarily aimed at reducing symptom burden and maintaining quality of life. However, a rapid deterioration in neurological and cognitive functioning may interfere with the oral intake of medication during the EOL phase [7]. Swallowing difficulties are seen in up to $85 \%$ of patients who are in the terminal phase of a malignant brain tumor [8]. Moreover, almost all brain tumor patients demonstrate progressive confusion or coma in the last days before death $[9,10]$. Thus, EOL symptoms may eventually hamper the administration 
of oral antiepileptic drugs (AEDs) [6]. To prevent inadequate AED treatment shortly before death, alternative AED treatment regimens are needed.

To overcome the difficulties in treating seizures during the EOL phase, our group has developed a guideline on the use of alternative administration routes of AEDs in glioma patients suffering from epilepsy that can easily be applied at home by patient's informal caregivers. In this guideline, we propose to start non-oral AED treatment with buccal clonazepam and intranasal midazolam as soon as the intake of oral AEDs is no longer possible $[11,12]$. Buccal clonazepam has the advantage of a long elimination half-life and can safely be applied as a prophylactic treatment in the out-of-hospital setting [13-15]. In the acute treatment of seizures, intranasal midazolam has shown a similar efficacy and safety in comparison with intravenous or rectal diazepam [16, 17]. Moreover, caregivers appear to prefer the use of intranasal midazolam $[12,18]$.

In the current study, we aim to assess the feasibility of a treatment protocol with the combination of buccal clonazepam and intranasal midazolam in the EOL phase of glioma patients according to an expert-based guideline. We evaluated the adherence to the study medication, caregiver's satisfaction, as well as seizure prevalence before and after initiation of the medication.

\section{Methods}

\section{Patients}

Adult patients ( $\geq 18$ years) with a histologically confirmed glioma (World Health Organization (WHO) grade II or III astrocytoma, oligodendroglioma, or oligoastrocytoma or WHO grade IV glioblastoma) in whom further antitumor therapy was considered to be no longer meaningful were eligible for inclusion. All patients had a history of at least one seizure during the course of their disease and received treatment with oral AEDs at study entry. An informal caregiver needed to be prepared to apply the treatment protocol at home. Patients with any known history of sensitivity to benzodiazepines or with a percutaneous endoscopic gastrostomy tube were excluded from the study. We considered all patients for participation who received routine follow-up at two tertiary referral centers for brain tumor patients in The Netherlands: one large community hospital (Medical Center Haaglanden, The Hague) and one university hospital (VU University Medical Center, Amsterdam). The study received approval from the Institutional Review Board (IRB) of both hospitals. All patients and caregivers provided written, informed consent.

\section{Study design}

We recruited patients at the outpatient clinic in both hospitals between May 2012 and November 2014. In case the patient decided to participate, the clinical nurse specialist instructed the patient and informal caregiver on the details of the protocol. We informed the patient's general practitioner on the patient's participation by letter. Patients who fulfilled the in- and exclusion criteria but declined to participate were recorded separately.

As soon as a patient was included in the study, the clinical nurse specialist contacted the patient and/or informal caregiver on a weekly basis and asked for the ability to use the regular oral AEDs. We instructed the patient or caregiver to continue the regular oral AED treatment as long as possible. We asked them to contact the nurse specialist in case swallowing difficulties were developing that hampered the regular oral intake of AEDs.

The treatment guideline was formed in 2011 during meetings at the VUmc with experts from different disciplines: neuro-oncology, pharmacology, epilepsy, and palliative medicine. Their main goal was to prepare a guideline to treat epilepsy in the EOL phase of brain tumor patients that could easily be applied by patient's caregivers in an out-of-hospital setting. After discussing possible non-oral AEDs that could be administered during the EOL phase, a combination of prophylactic treatment with buccal clonazepam and emergency treatment with intranasal midazolam was regarded as the most appropriate AED treatment regimen.

The content of the guideline was as follows: when the administration of oral AEDs was no longer possible, the caregiver started to administer prophylactic treatment with buccal clonazepam with an initial dose of two-four times $0.5 \mathrm{mg} /$ day, followed by a maintenance dose of four times $0.5-1.0 \mathrm{mg}$ /day. In case of recurrent seizures, the daily dose was gradually increased in steps of 1-2 mg/day until seizures were controlled, intolerable adverse effects occurred, or the maximum maintenance dose of $20 \mathrm{mg} /$ day had been reached. Dose adjustments were made after consultation of the nurse specialist or treating physician. Clinically visible seizures were treated with one puff of intranasal midazolam in each nostril (total of $5 \mathrm{mg}$ midazolam). If seizures persisted for $5 \mathrm{~min}$ despite the administration of intranasal midazolam, a second dose of $5 \mathrm{mg}$ was administered. We instructed the caregiver to contact the hospital or general practitioner in case a second dose of midazolam failed as well. We considered the emergency treatment successful in case seizures were suppressed for at least $2 \mathrm{~h}$ after administration of the last dose.

The nurse specialist stayed in contact with the caregiver at least once a week as soon as the study medication (buccal clonazepam and/or intranasal midazolam) was started, to collect data on seizure prevalence, use of the study medication, its efficacy, and possible adverse effects. In case of recurrent 
seizures, the nurse specialist contacted the caregiver on a daily basis until seizure control was effected. We derived additional information on various aspects of the EOL phase from a short questionnaire, completed by the caregivers after patient's death. The questionnaire included questions on the patient's general condition during the EOL phase; reasons for nonadministration of the study medication; adverse drug effects; as well as three questions on caregiver's satisfaction with (1) explanation of the treatment protocol, (2) the administration of buccal clonazepam, and (3) the administration of intranasal midazolam. We collected data on demographics, tumor histology, seizure type, and seizure frequency before the EOL phase from the hospital medical charts.

\section{Study end points}

The primary end point of the pilot study was adherence to the treatment protocol, determined by the number of patients that were treated according to the study protocol until death. The treatment protocol was deemed feasible if at least $50 \%$ of the included patients in whom oral AED treatment was no longer possible were treated according to the guideline. The secondary end points were caregiver's satisfaction with the study medication (according to a 7-point Likert scale ranging from (1) completely dissatisfied to (7) completely satisfied), seizure prevalence after initiation of the study medication, adverse effects, and the number of hospital admissions due to treatment failure.

\section{Results}

\section{Patients}

Of the 34 patients who were approached, 25 patients gave consent to participate (Fig. 1). At the end of the study, 23 out of 25 patients $(92.0 \%)$ had died. The median duration from time of consent to time of death was 3.2 months for these 23 patients (range 0.3-7.2 months). Four of 23 patients $(17.4 \%)$ were able to use their oral AEDs until death. Twenty of 23 caregivers (response rate $87.0 \%$ ) completed the questionnaire on the EOL phase with a median interval of 7.5 months (range 1-20 months) after patient's death.

A summary of baseline data of all patients is outlined in Table 1 (for details per patient, see supplementary Table 1). Seventeen of 23 patients $(73.9 \%)$ were male with a mean age of 56.7 years. Nineteen of 23 patients $(82.6 \%)$ died at home. Twelve of 23 patients $(52.2 \%)$ had a history of generalized seizures, 7 patients $(30.4 \%)$ of simple partial seizures, 3 (13.0\%) of a combination of partial and generalized seizures, and 1 patient $(4.3 \%)$ of complex partial seizures. Fifteen of 23 patients $(65.2 \%)$ had a seizure frequency of $<1$ seizure/month before the start EOL phase, the other 8 patients $(37.5 \%)$ reported higher frequencies.

\section{Treatment}

Fourteen of 19 patients $(73.7 \%)$ used either buccal clonazepam or intranasal midazolam or both medications. One of

Fig. 1 Inclusion of subjects

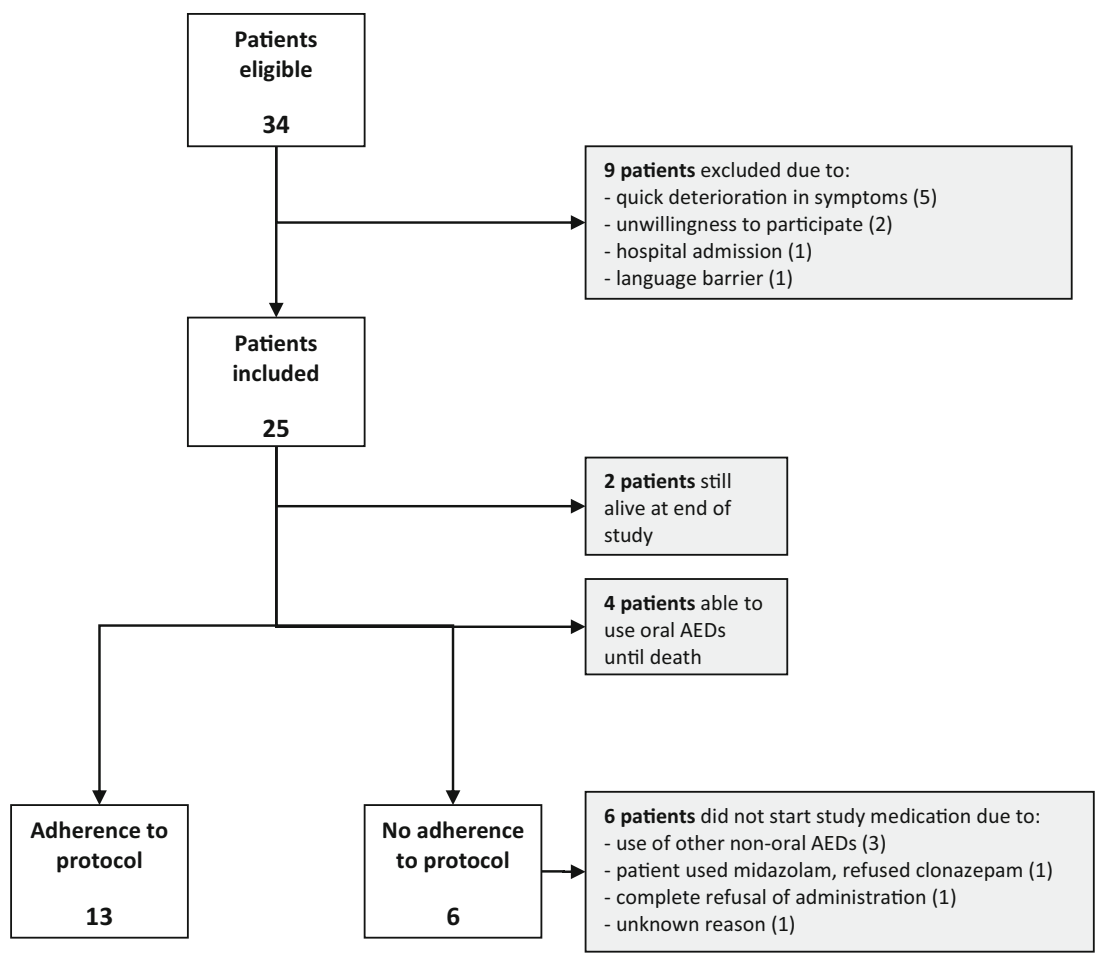


Table 1 Baseline characteristics

\begin{tabular}{|c|c|c|}
\hline & & $N(\%)$ \\
\hline \multirow[t]{2}{*}{ Gender } & Male & $17(73.9)$ \\
\hline & Female & $6(26.1)$ \\
\hline Mean age, years (SD) & & $56.7(8.5)$ \\
\hline \multirow[t]{3}{*}{ Tumor grade } & Glioblastoma & $13(56.5)$ \\
\hline & Anaplastic glioma & $6(26.1)$ \\
\hline & Low-grade glioma & $4(17.4)$ \\
\hline \multirow[t]{2}{*}{ Primary caregiver } & Partner & $22(95.7)$ \\
\hline & Professional caregiver & $1(4.3)$ \\
\hline \multirow[t]{4}{*}{ Seizure type } & Generalized & $12(52.2)$ \\
\hline & Simple partial & $7(30.4)$ \\
\hline & Complex partial & $1(4.3)$ \\
\hline & Both partial and generalized & $3(13.0)$ \\
\hline \multirow[t]{3}{*}{ Seizure frequency before EOL phase } & $<1 /$ month & $15(65.2)$ \\
\hline & $>1 /$ month & $6(26.1)$ \\
\hline & $>1 /$ week & $2(8.7)$ \\
\hline KPS at 1 week before death, mean (range) & & $30(20-50)$ \\
\hline \multirow[t]{3}{*}{ Place of death } & Home & $19(82.6)$ \\
\hline & Hospice & $3(13.0)$ \\
\hline & Care hotel & $1(4.3)$ \\
\hline \multirow[t]{3}{*}{ Adherence to study protocol } & Yes & $13(56.5)$ \\
\hline & No & $6(26.1)$ \\
\hline & Use of oral AEDs until death & $4(17.4)$ \\
\hline \multirow[t]{2}{*}{ Use of buccal clonazepam $(n=19)$} & Yes & $13(68.4)$ \\
\hline & No & $6(31.6)$ \\
\hline \multirow[t]{5}{*}{ Start date buccal clonazepam, days before death $(n=13)$} & $>14$ & $2(15.4)$ \\
\hline & $7-14$ & $2(15.4)$ \\
\hline & $3-7$ & $2(15.4)$ \\
\hline & $1-3$ & $5(38.5)$ \\
\hline & 1 & $2(15.4)$ \\
\hline \multirow[t]{2}{*}{ Use of intranasal midazolam $(n=19)$} & Yes & $6(31.6)$ \\
\hline & No & $13(68.4)$ \\
\hline
\end{tabular}

these 14 patients only received intranasal midazolam, as he refused the administration of buccal clonazepam. So eventually, 13 of 19 patients (68.4\%; $95 \%$ CI 47.1-89.7\%) who had developed swallowing difficulties completely adhered to the study protocol. In the remaining $6 / 19$ patients $(35.2 \%)$, the study medication was not administered for various reasons (Fig. 1). One of these six patients who did not receive the study medication according to the treatment protocol reported seizures during the last week before death. In all 14 patients who used any study medication, the caregiver completed a questionnaire on the EOL phase. Treatment characteristics per patient are outlined in supplementary Table 2 .

Thirteen patients used buccal clonazepam as prophylactic treatment. After the start of buccal clonazepam, 8 of 13 patients $(61.5 \%)$ remained seizure free. The other five patients reported seizures after the start of clonazepam, which all occurred during the last week before death. In 2 of 13 patients
(15.4\%), a dose increase was necessary due to recurrent seizures. In the remaining 3 of 13 patients (23.1\%), a dose increase was not necessary, as seizure control was achieved after emergency treatment with intranasal midazolam. Treatment with buccal clonazepam was started during the last week before death in 9 of 13 patients (69.2\%).

Six patients received treatment with intranasal midazolam at least once. In these 6 patients, a total of 16 seizures were treated, and only in 1 of these 16 seizures a second dose was necessary to achieve seizure control. One of the six patients who received intranasal midazolam refused prophylactic treatment with buccal clonazepam. In all six patients who were treated with intranasal midazolam, seizure control could be reached.

None of the patients needed to be transferred to the hospital due to recurrent seizures. In one patient, the general practitioner decided to replace buccal clonazepam by subcutaneous 
midazolam 3 days before death due to seizure recurrence. Adverse effects of the study medication were scarce. In one patient, a dry mouth was reported after start of the study medication. In none of the patients intolerable adverse effects were reported that led to discontinuation of treatment or that withheld the physician from increasing the clonazepam maintenance dose.

\section{Caregiver's satisfaction}

Of all 20 caregivers who completed the questionnaire on the EOL phase, 19 caregivers $(95.0 \%)$ had the opinion that they had received clear instructions by the nurse specialist on the use of the study medication. None of the 13 caregivers who had administered buccal clonazepam were dissatisfied with the use of the medication. Six of 13 caregivers (46.2\%) were mostly or completely satisfied with its use ( 6 or 7 on a 7 -point Likert scale) and 7 of $13(53.8 \%)$ were neutral or somewhat satisfied (4 or 5 out of 7). Of the six caregivers who had administered intranasal midazolam, all were mostly or completely satisfied with its use.

\section{Discussion}

The primary objective of this study was to assess the feasibility of a non-oral AED treatment protocol in the EOL phase of glioma patients. According to our predefined criteria, it appears to be feasible to administer AED treatment with buccal clonazepam and intranasal midazolam shortly before death, as approximately two thirds of patients who had developed swallowing difficulties showed adherence to the study medication regimen. Moreover, we found that all caregivers were at least to some extent satisfied with the administration of buccal clonazepam or intranasal midazolam.

This is the first study in which the feasibility of non-oral AED treatment has been prospectively examined in the EOL phase of glioma patients. Our results confirm the high prevalence of dysphagia and loss of consciousness that underlie swallowing difficulties in the EOL phase [9]. In line with previous retrospective studies, we found that only $17 \%$ of patients were able to use their regular oral AEDs until death [3]. To date, no information has been available on the time at which EOL symptoms start to hamper oral drug administration in glioma patients. Our study reveals that swallowing difficulties become burdensome at a relatively late stage of the disease. In $69 \%$ of patients who used the study medication, swallowing difficulties did not develop until the last week before death.

Almost all participating caregivers showed satisfaction with the use of the non-oral AED treatment, particularly regarding the emergency treatment with intranasal midazolam. In patients with refractory non-tumor-related epilepsy, caregiver's satisfaction with the use of nasal spray has also been demonstrated [18]. The absence of any transfers to the hospital due to uncontrolled seizures as well as the lack of serious side effects may have contributed to the caregiver's satisfaction. In addition, in only $6 \%$ of cases the caregiver or patient refused participation in the study. The clear willingness to participate seems to reflect an urgent need to prevent seizure recurrence in the EOL phase. As it is known that caregivers particularly fear seizures shortly before death, they probably find it reassuring to be able to manage the seizures when necessary [19].

Altogether, $26 \%$ of patients who were included in the study reported at least one seizure during the last week before death. These findings correspond to previous series that showed seizure prevalence between 28 and $38 \%$ during the last week $[6,8,20]$. However, we were unable to determine the true efficacy of the study medication given the absence of an appropriate control group in our study. Other limitations of the study include the relatively small sample size and the high dependence on inexperienced caregivers who had a central role in administering the medication, observing its effect, as well as reporting information to the nurse specialist. Furthermore, adverse drug effects were rarely reported and may have gone unnoticed due to the patient's rapidly declining condition. In addition, the role of prophylactic treatment in patients without a history of seizures is still unknown.

Despite the fact that five patients still experienced seizures after treatment with prophylactic buccal clonazepam had been started, seizure freedom or at least temporary seizure control could eventually be achieved in all patients. However, a randomized controlled study is required to know whether the study medication leads to a reduction in seizures during the EOL phase. It is noteworthy that none of the participating patients needed rehospitalization due to refractory seizures. A previous study demonstrated that avoiding transitions between health care settings is of major importance in glioma patients and may eventually contribute to a more dignified death [21].

In conclusion, the results of this study demonstrate that it is feasible to treat seizures in the EOL phase according to a treatment protocol with a combination of non-oral benzodiazepines. It seems to provide an important level of comfort among caregivers to be able to manage seizures in an out-ofhospital setting. As the risk of seizures persists until death, AED treatment should be easily accessible to all glioma patients in the EOL phase who have a history of epilepsy, particularly when EOL symptoms start to interfere with their oral AED intake.

Acknowledgments We are very grateful to the patients and their informal caregivers who took part in this study. We particularly thank the 
clinical nurse specialists Hanneke Zwinkels, Ellen Gortmaker, Claudia Nijboer, and Alieke Weerdesteijn for their assistance in coordinating the study.

Funding J.A.F. Koekkoek and M.J.B. Taphoorn received funds from the St. Jacobusstichting The Hague, foundation "ZOLEON," and foundation "Chanrone."

Conflict of interest The authors declare that they have no competing interests.

Open Access This article is distributed under the terms of the Creative Commons Attribution-NonCommercial 4.0 International License (http:// creativecommons.org/licenses/by-nc/4.0/), which permits any noncommercial use, distribution, and reproduction in any medium, provided you give appropriate credit to the original author(s) and the source, provide a link to the Creative Commons license, and indicate if changes were made.

\section{References}

1. de Groot M, Reijneveld JC, Aronica E, Heimans JJ (2012) Epilepsy in patients with a brain tumour: focal epilepsy requires focused treatment. Brain 135:1002-1016

2. Kerkhof M, Vecht CJ (2013) Seizure characteristics and prognostic factors of gliomas. Epilepsia 54(Suppl 9):12-17

3. Pace A, Villani V, Di LC et al (2013) Epilepsy in the end-of-life phase in patients with high-grade gliomas. J Neurooncol 111:83-86

4. Baldwin K, Miller L, Scott JB (2002) Proactive identification of seizure risk improves terminal care. Am J Hosp Palliat Care 19: 251-258

5. Koekkoek JA, Dirven L, Reijneveld JC, Postma TJ, Grant R, Pace A (2014) Epilepsy in the end of life phase of brain tumor patients: a systematic review. Neuro Oncol Pract 1(3):134-140

6. Sizoo EM, Koekkoek JA, Postma TJ et al (2014) Seizures in patients with high-grade glioma: a serious challenge in the end-of-life phase. BMJ Supp Palliat Care 4:77-80

7. Pace A, Di LC, Guariglia L, Jandolo B, Carapella CM, Pompili A (2009) End of life issues in brain tumor patients. J Neurooncol 91: $39-43$
8. Sizoo EM, Braam L, Postma TJ et al (2010) Symptoms and problems in the end-of-life phase of high-grade glioma patients. Neuro Oncol 12:1162-1166

9. Walbert T, Khan M (2014) End-of-life symptoms and care in patients with primary malignant brain tumors: a systematic literature review. J Neurooncol 117:217-224

10. Sizoo EM, Pasman HR, Dirven L et al (2014) The end-of-life phase of high-grade glioma patients: a systematic review. Support Care Cancer 22:847-857

11. Koekkoek JA, Boddaert MS, Taphoorn M (2014) Gliomas: fighting until the end against epilepsy; administration of antiepileptic drugs in the end-of-life phase. Ned Tijdschr Geneeskd 158:A6924

12. Anderson GD, Saneto RP (2012) Current oral and non-oral routes of antiepileptic drug delivery. Adv Drug Deliv Rev 64:911-918

13. Browne TR (1976) Clonazepam. A review of a new anticonvulsant drug. Arch Neurol 33:326-332

14. Schols-Hendriks MW, Lohman JJ, Janknegt R, Korten JJ, Merkus FW, Hooymans PM (1995) Absorption of clonazepam after intranasal and buccal administration. Br J Clin Pharmacol 39:449-451

15. Crevoisier C, Delisle MC, Joseph I, Foletti G (2003) Comparative single-dose pharmacokinetics of clonazepam following intravenous, intramuscular and oral administration to healthy volunteers. Eur Neurol 49:173-177

16. Lahat E, Goldman M, Barr J, Bistritzer T, Berkovitch M (2000) Comparison of intranasal midazolam with intravenous diazepam for treating febrile seizures in children: prospective randomised study. BMJ 321:83-86

17. Bhattacharyya M, Kalra V, Gulati S (2006) Intranasal midazolam vs rectal diazepam in acute childhood seizures. Pediatr Neurol 34: 355-359

18. de Haan GJ, van der Geest P, Doelman G, Bertram E, Edelbroek P (2010) A comparison of midazolam nasal spray and diazepam rectal solution for the residential treatment of seizure exacerbations. Epilepsia 51:478-482

19. Bausewein C, Hau P, Borasio GD, Voltz R (2003) How do patients with primary brain tumours die? Palliat Med 17:558-559

20. Flechl B, Ackerl M, Sax C et al (2013) The caregivers' perspective on the end-of-life phase of glioblastoma patients. J Neurooncol 112: 403-411

21. Sizoo EM, Taphoorn MJ, Uitdehaag B et al (2013) The end-of-life phase of high-grade glioma patients: dying with dignity? Oncologist 18:198-203 